\title{
Dermatology Archives
}

\section{Excellent Response to Hydroxychloroquine in a Young Man with Lupus Erythematosus Panniculitis}

\section{Aníbal María Parigini',", María Clara de Diego ${ }^{1}$, Julieta Ruiz Beguerie ${ }^{1}$, Victoria Duran Paredes $^{2}$ and Javier Anaya ${ }^{2}$}

\author{
${ }^{1}$ Department of Dermatology, Austral University Hospital, Argentina \\ ${ }^{2}$ Department of Pathology, Austral University Hospital, Argentina
}

\begin{abstract}
Lupus erythematosus panniculitis is a rare variant of lupus erythematosus. We present the case of a young man with ulcerative colitis treated with mesalazine and a history of recurrent painful and indurated lesions on his face. A diagnosis of lupus profundus was made taking in account the clinical manifestations and the histological and immunofluorescence findings. An excellent initial response and stabilization of the dermatosis was achieved with a combined treatment with antimalarial drugs and systemic corticosteroids.

Keywords

Lupus, Lupus erythematosus panniculitis, Lupus profundus, Panniculitis, Hydroxychloroquine

Abbreviations

LEP: Lupus Erythematosus Panniculitis; LE: Lupus Erythematosus; DLE: Discoid Lupus Erythematosus; SLE: Systemic Lupus Erythematosus; SCTCL: Subcutaneous T-Cell Lymphoma; CGD-TCL: Gamma-Delta T-Cell Lymphoma; MRI: Magnetic Resonance Imaging; TCR: T-Cell Receptor
\end{abstract}

\section{Introduction}

Lupus erythematosus panniculitis (LEP) is a rare form of chronic lupus erythematosus (LE) [1,2]. It occurs in $1-3 \%$ of patients with cutaneous LE [2]. It can be the only manifestation of this entity or it may appear in patients with discoid lupus erythematosus (DLE) or systemic lupus erythematosus (SLE) [1,2].

Most authors also use the term lupus profundus (LP) as a synonym, although some make a distinction between them, arguing that LEP refers to the solely involvement of the subcutaneous tissue, whereas LP also involves the dermis [3]. LEP affects both genders with female predominance. The median age of presentation is 41 years, with a range from 30 to 60 years [1].

\section{Case Presentation}

A 35-year-old male patient with a personal history of ulcerative colitis treated with mesalazine and an alleged history of lichen planus on hands, ears and scalp treated with meprednisone at a dose of $20 \mathrm{mg}$ daily for approximately ten years consulted the dermatology department for presenting recurrent painful and indurated lesions on his face. He reported that these lesions had developed a year before the consultation. He referred that the dermatosis had begun as a single lesion on his left cheek that subsided spontaneously. Subsequently, he presented a relapse in the same location and afterwards he developed indurated areas in both parotid regions.

Physical examination showed scars in both auricles with atrophy of the left lobe, a scar on the dorsum of the nose dorsum and tender nodules were evidenced on both cheeks, without changes on the overlying skin. Additionally, he presented submaxillary, bilateral, elastic, and mo-

\footnotetext{
*Corresponding author: Aníbal María Parigini, Department of Dermatology, Austral University Hospital, Av Juan Domingo Perón 1500, Pilar, B16290DT, Provincia de Buenos Aires, Argentina, E-mail: aparigin@cas.austral.edu.ar

Received: May 29, 2018: Accepted: July 03, 2018; Published online: July 05, 2018

Citation: Parigini AM, de Diego MC, Ruiz Beguerie J, et al. (2018) Excellent Response to Hydroxychloroquine in a Young Man with Lupus Erythematosus Panniculitis. Dermatol Arch 2(1):51-54
} 
Citation: Parigini AM, de Diego MC, Ruiz Beguerie J, et al. (2018) Excellent Response to Hydroxychloroquine in a Young Man with Lupus Erythematosus Panniculitis. Dermatol Arch 2(1):51-54

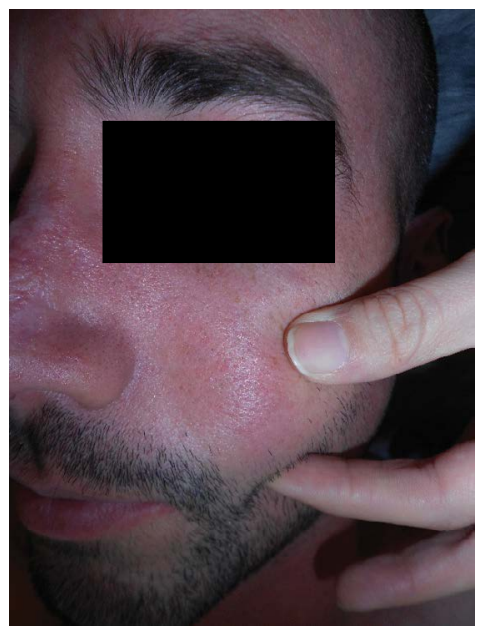

Figure 1: Tender nodule evidenced in left cheek.

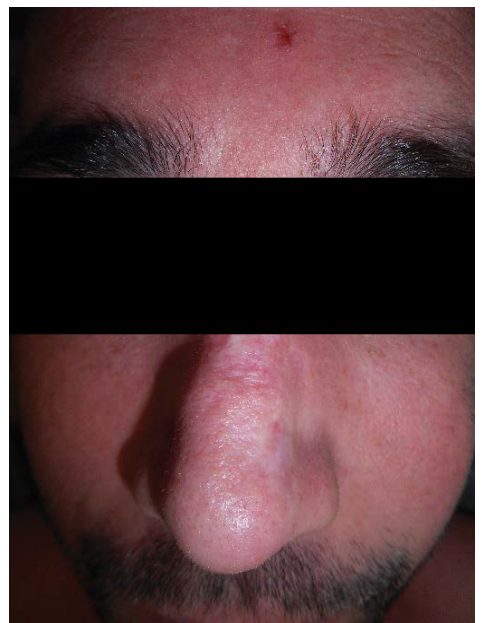

Figure 2: Scar in the nose dorsum.

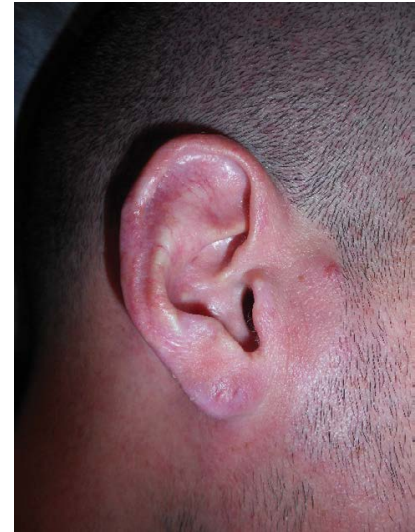

(a)

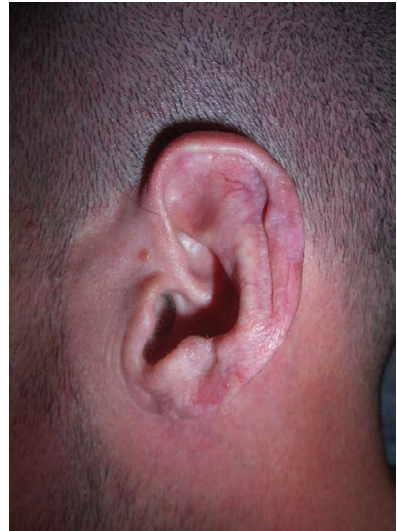

(b)
Figure 3: a,b) Scars in both ears and atrophy of the left lobe.

bile adenopathies (Figure 1, Figure 2 and Figure 3).

Given this clinical picture, the diagnosis of lupus panniculitis was considered.

A magnetic resonance imaging (MRI) with contrast

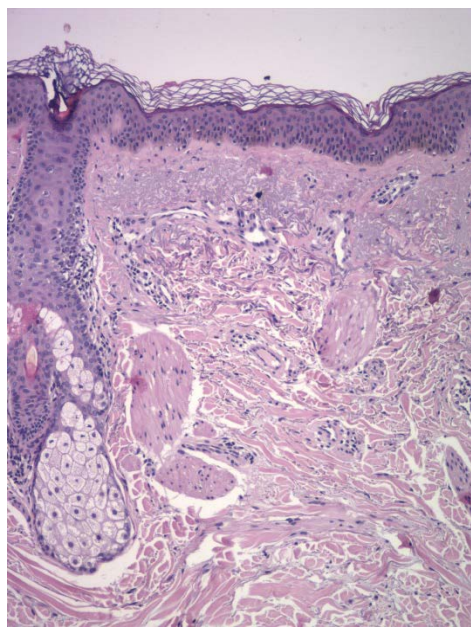

Figure 4: Epidermis with no significant alterations

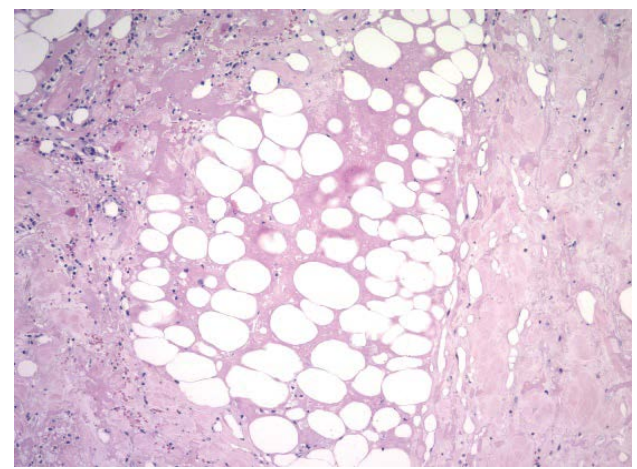

Figure 5: Degenerative-fibrinoid changes of collagen, eosinophilia between adipocytes with necrosis and local lipophagy.

of the head and neck area was performed, which showed thickening of the integuments with foci of hyperintense signals in STIR and T2 in both cheeks. Additionally, hypointense nodular formations within the subcutaneous fat were evident in T1 and moderately intense in T2.

A biopsy of one of the lesions was performed, and it demonstrated a moderate perivascular and periappendageal inflammatory infiltrate composed of lymphocytes accompanied by plasma cells within the deep dermis and extending into the subcutaneous fat with involvement of lobules and septa. Furthermore, it exhibited hyaline fat necrosis with foci of lipophagy and fibrinoid degenerative changes of collagen. There were no significant changes in the epidermis. With these findings, the pathologist suggested the diagnosis of lupus profundus. To confirm this, a direct immunofluorescence performed on non-affected skin revealed IgG, C1q and C3 deposits at the dermoepidermal junction (Figure 4, Figure 5 and Figure 6).

Additional laboratory studies showed positive antinuclear antibodies with a titre of $1 / 1280$ with homogeneous pattern. On the other hand, he did not exhibit anti-dou- 
Citation: Parigini AM, de Diego MC, Ruiz Beguerie J, et al. (2018) Excellent Response to Hydroxychloroquine in a Young Man with Lupus Erythematosus Panniculitis. Dermatol Arch 2(1):51-54

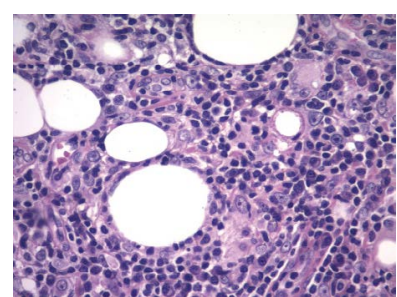

(a)

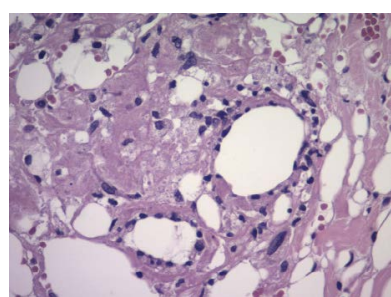

(b)
Figure 6: $a, b)$ Moderate lymphocytic infiltrates with plasmocytes.

ble-stranded DNA antibodies, anti SCL 70, anti-Smith, anti Ro, anti La, anti-cardiolipin, or rheumatoid factor.

The final diagnosis was LEP. The patient started therapy with hydroxychloroquine $400 \mathrm{mg}$ orally daily and the interruption of meprednisone was planned according to the evolution of the dermatosis.

After 4 weeks of treatment with meprednisone $20 \mathrm{mg}$ and hydroxychloroquine $400 \mathrm{mg}$ daily, the patient presented an excellent initial response with marked decrease in nodules' size and pain. However, there was an exacerbation of the ulcerative colitis, so, despite there is no interaction described between antimalarials and ulcerative colitis, it was decided to reduce hydroxychloroquine to $200 \mathrm{mg}$ daily and continue with meprednisone at $20 \mathrm{mg}$ daily.

The patient has been under this treatment for nine months with only one episode of new lesions, which was controlled with an increase in the dose of meprednisone to $40 \mathrm{mg}$ daily for 3 weeks. With a stabilization of the dermatosis.

\section{Discussion}

LEP has a chronic recurrent course. Patients generally present with tender deep subcutaneous nodules or plaques. The overlying skin can be normal or present DLE lesions. When the lesions withdraw, they leave swelling and atrophy $[1,4]$.

In a series of 17 patients, the authors found that $47.5 \%$ presented troughs suggesting lipoatrophy, the same percentage showed erythema and $41.2 \%$ had subcutaneous nodules. Additionally, one patient developed scarring alopecia [2].

The lesions usually involve the face, scalp, the upper arms, face, trunk, breasts, buttocks and thighs [1-3]. There have been described lesions that developed on sites of trauma or injections $[3,5]$. And there can be aggravation at the place where the biopsy is performed [4].

Although laboratory findings are often normal, antinuclear antibodies can be positive, generally showing a titre of 1/80 or less. Positive anti dsDNA may be observed at a lower frequency. Other abnormalities include anemia, thrombocytopenia and leukopenia [1-3]. The case we present exhibited an unusually high titre.

In approximately half of the cases, histopathologic changes are confined to the subcutaneous tissue, showing a mostly lobular panniculitis with an infiltrate predominantly lymphocytic. Lymphoid follicles are a characteristic feature, often showing germinal centers with numerous plasma cells at the periphery $[4,6]$. These are not pathognomonic as they have been described in other diseases, such us deep morphea, erythema nodosum, dermatomyositis, and erythema induratum of Bazin, but they are quite useful as they are not seen in subcutaneous lymphomas $[2,4]$. There is also hyaline fat necrosis. Some lymphocytes may show nuclear dust, which is rare in other forms of panniculitis. The septa appear to be sclerotic and hyaline with lymphocytes and plasma cells [4].

The other half of cases described in the literature evidenced there are also epidermal changes - atrophy, vacuolar interface, thickened basement membrane - and dermal alterations - interstitial mucin between the bundles of collagen and superficial and deep perivascular infiltrate of lymphocytes - of DLE [4].

The immunofluorescence shows linear deposition of IgM and C3 along the dermoepidermal junction [4].

The main differential diagnosis to take into account is a subcutaneous T-Cell lymphoma (SCTCL), which presents similar lesions on the same areas affected by LEP, though the latter affects more frequently the lower limbs. SCTCL may also be associated with an autoimmune disorder. They both share certain histologic findings $[7,8]$. Some have proposed that LEP and SCTCL are part of a spectrum of the same disease given an overlap in the clinical and histological findings. In LEP there is a mixed population of CD4 and CD8 and, in most cases, the T-cell receptor (TCR) gene rearrangement studies show a polyclonal pattern. It is rare to see lymphocytes with atypia. In SCTCL there are multiple painless plaques or nodules rarely ulcerated on the trunk or lower limbs. Lymphocytes show atypia with variable sizes and irregular nuclear contours showing polylobation and blebs. There is a monoclonal pattern in the TCR gene rearrangement studies and the immunohistochemistry shows a CD8-positive and CD4-negative cytotoxic phenotype. SCTCL shows an alfa/beta T cell phenotype and must be distinguished from the cutaneous gamma-delta T-cell lymphoma (CGD-TCL) given that the first one shows a remarkably better prognosis. CGDTCL has a 5 -year overall survival rate of $11 \%$. It presents with ulcerated nodules and plaques affecting mainly the lower limbs. There are atypical lymphocytes from the subcutaneous tissue to the epidermis with extensive necrosis and apoptosis and the immunohistochemistry is negative for CD4 and CD8 [8]. 
Citation: Parigini AM, de Diego MC, Ruiz Beguerie J, et al. (2018) Excellent Response to Hydroxychloroquine in a Young Man with Lupus Erythematosus Panniculitis. Dermatol Arch 2(1):51-54

It is of outmost importance to recognize a SCTCL since it presents a greater morbidity as well as a risk for hemophagocytic lymphohistiocytosis. When there is mild lymphocyte atypia and a monoclonal TCR gene rearrangement it may be difficult to make a definitive distinction and some authors have proposed the term atypical lymphocytic lobular panniculitis $[7,8]$.

Antimalarial drugs are the first line of therapy of LEP. They are generally combined with systemic steroids in the beginning because the former tend to take effect slowly and they may even take up to 3 months to show results. The usual doses for hydroxychloroquine and chloroquine are $200-400 \mathrm{mg} /$ day and $250-500 \mathrm{mg}$ orally respectively $[1,3]$.

Most patients respond to these drugs, but skin lesions recur after interrupting therapy, with a median of 10 months (range of 1-50 months) [2]. Some authors mention thalidomide as the most effective treatment for LEP at a dose of $100 \mathrm{mg} /$ day [1].

Oral corticosteroids can be used, especially when LEP is associated with LES. Topical use of this group of drugs can be useful, but intralesional injections may exacerbate the atrophy and are not effective [1].

This patient is under treatment with a combination of hydroxychloroquine $200 \mathrm{mg} /$ day and meprednisone 20 $\mathrm{mg} /$ day with sustained response.

Other treatments that have been described in case reports include sulfone, azathioprine, mycophenolate mofetil, methotrexate, cyclosporine, dapsone, and endovenous immunoglobulin [1-3]. A recent case report described a patient refractory to most therapies that was treated with rituximab with a good response and without recurrence [9].

\section{Conclusions}

LEP is an infrequent manifestation of lupus and its diagnosis can be challenging. It is important to differentiate it from similar manifesting cutaneous lymphomas.

We decided to report a case of a young man with LEP and ulcerative colitis to describe the steps necessary to reach the diagnosis and to show the excellent response to the first line of treatment.

\section{Acknowledgments}

Laboratory of Dr. Roberto Iotti for performing the direct immunofluorescence.

\section{References}

1. Fraga J, García Díez A (2008) Lupus erythematosus panniculitis. Dermatol Clin 26: 453-463.

2. Park HS, Choi JW, Kim B, et al. (2010) Lupus erythematosus panniculitis: Clinicopathological, immunophenotypic, and molecular studies. Am J Dermatopathol 32: 24-30.

3. Castrillón MA, Murrell DF (2017) Lupus profundus limited to a site of trauma: Case report and review of the literature. Int J Women's Dermatology 3: 117-120.

4. Requena L, Yus ES (2001) Panniculitis Part II. Mostly lobular panniculitis. J Am Acad Dermatol 45: 325-361.

5. Choi JY, Kim HS, Lee G-Y (2017) Case of lupus erythematosus panniculitis triggered by human papillomavirus quadrivalent vaccine injection. J Dermatol 44: 1420-1421.

6. Wick MR (2017) Panniculitis: A summary. Semin Diagn Pathol 34: 261-272.

7. Magro CM, Crowson AN, Kovatich AJ, et al. (2001) Lupus profundus, indeterminate lymphocytic lobular panniculitis and subcutaneous T-cell lymphoma: A spectrum of subcuticular T-cell lymphoid dyscrasia. J Cutan Pathol 28: 235247.

8. Shiau CJ, Daoud MSA, Wong SM, et al. (2015) Lymphocytic panniculitis: An algorithmic approach to lymphocytes in subcutaneous tissue. J Clin Pathol 68: 954-962.

9. Prieto-Torres L, Alegría-Landa $\mathrm{V}$, Morales-Moya AL, et al. (2017) Lupus panniculitis refractory to multiple therapies treated successfully with rituximab: A case report and literature review. Australas J Dermatol 1: 1-2. 